\title{
COMPORTAMIENTO TEMPORAL DE LAS MITOSPORAS DE Cladosporium EN LA ATMOSFERA DE GALICIA (ESPAÑA)
}

\author{
(Temporary behaviour of Cladosporium mitospores in the atmosphere \\ of Galicia, Spain)
}

\author{
* M.J. Aira; **Rodríguez-Rajo, F.J. \& **Jato, V. \\ *Departamento de Botánica, Facultad de Farmacia, \\ Universidad de Santiago (España) \\ **Departamento de Biología Vegetal y Ciencias del Suelo, \\ Facultad de Ciencias de Ourense, Universidad de Vigo (España)
}

Palabras clave: Mitosporas fúngicas, atmósfera, Cladosporium, Galicia, España. Key words: Fungal mitospores, atmosphere, Cladosporium, Galicia, Spain.

\section{RESUMEN}

Se ha realizado el recuento de las mitosporas de poblaciones de Cladosporium en la atmósfera de ocho ciudades de Galicia (España), mediante un sistema de captación volumétrica no viable, obteniéndose un total de 40 registros anuales.

Los valores promedio de los años de estudio oscilaron entre 96.726 mitosporas en Coruña y 326.898 en Trives, destacando en todas las localidades una mayor abundancia de las mismas durante la época estival. El máximo diario de Cladosporium cladosporioides se registró en Ourense (7.556 mitosporas $/ \mathrm{m}^{3}$ el 8 de septiembre de 2004) mientras que el de Cladosporium herbarum correspondió a Trives (7.130 mitosporas $/ \mathrm{m}^{3} \mathrm{el}$ 8 de julio de 2003). El modelo de comportamiento intradiario varió según la localidad, pero en general se observó una mayor concentración de mitosporas a partir de las $13 h$.

\section{INTRODUCCION}

La presencia de mitosporas de hongos en la atmósfera está ampliamente documentada en distintas partes del mundo (Airaudi \& Marchisio, 1997; Chakraborty et al., 2001; Aira et al., 2002; Antoni et al., 2004; Noé et al., 2006). Muchas son las aplicaciones de este tipo de estudios, desde aquellas que se orientan a la prevención de plagas en los cultivos (Rodríguez et al.,

\begin{abstract}
By using a non viable volumetric capturing system it was possible to count populations of Cladosporium mitospores in the atmosphere of eight cities of Galicia (Spain), yielding a total of 40 annual records.

Average values of the examined years ranged from 96.726 mitospores in Coruña and 326.898 in Trives, the highest abundance occurring during summer in all the localities. The daily maximum of Cladosporium cladosporioides was recorded in Ourense (7.556 mitospores/m on September 2004) while that of Cladosporium herbarum was detected in Trives (7.130 mitospores/m on july 8 2003). The intradaily model of behaviour varied according to the locality yet in general a major mitospore concentration could be observed since one óclock p.m.
\end{abstract}

2002; Albelda et al., 2005), las que procuran la conservación de monumentos y obras de valor histórico-artístico (Montacutelli et al., 2000; Maggi et al., 2000), o aquellas cuyo interés se relaciona con los problemas de tipo clínico, por las alergias respiratorias que pueden producir sus esporas (Dale et al, 2000; Oliveira et al., 2006).

Basándonos en el gran interés científico y social de estos aspectos, la Red Gallega de Aerobiología (R.G.A.), formada por investigadores de las Universidades de Santiago y Vigo (España) desarrolla desde el año 1993 
una línea de investigación en Aeromicología, dedicándose a recoger, identificar y contabilizar de forma periódica las mitosporas más abundantes en la atmósfera de diferentes localidades gallegas.

Cladosporium (Link ex Fr.), es un hongo cosmopolita que incluye a numerosas especies que pueden vivir en el suelo y sobre gran variedad de sustratos (lana, papel, semillas, restos vegetales, etc.), de ahí que haya sido citado como uno de los hongos cuyas mitosporas o conidios son muy abundantes en la atmósfera, principalmente en los ambientes externos. Junto con Alternaria es uno de los hongos utilizados por la industria farmacéutica en el diagnóstico y tratamiento de las alergias respiratorias, cultivándolo a gran escala para producir biomasa que sirva de materia prima para la preparación de los extractos estandarizados. Sus mitosporas presentan un marcado carácter alergénico y se le ha implicado en casos de asma y rinitis (Vijay et al., 1991; D’Amato \& Spieksma, 1995; Myszkowska et al., 2002).

En las muestras aerobiológicas obtenidas por la R.G.A., son muy abundantes dos tipos de mitosporas pertenecientes a este género, pero fáciles de diferenciar entre sí al microscopio óptico de acuerdo con el trabajo realizado por Mediavilla et al. (1997), quienes proponen realizar el recuento de las que corresponden a Cladosporium cladosporioides (Fr.) de Vries y especies afines, separándolas de las que pertenecen a Cladosporium herbarum (Pers.) Link ex Gray y especies afines.

En este trabajo se presentan los resultados obtenidos para Cladosporium en 8 ciudades de Galicia entre los años 1993 y 2005.

\section{MATERIALES Y METODOS}

El presente estudio se llevó a cabo entre los años 1993 y 2005, en ocho localidades de Galicia, en el N.O. de España (Ourense, Santiago, Coruña, Viveiro, Lugo, Vigo, Verín y Trives), seleccionadas por ser los mayores núcleos de población y por recoger la variabilidad biogeográfica de la región gallega (Figura 1). Estas muestras se registraron de la siguiente manera: durante 10 años en Ourense, 9 años en Santiago, 6 años en Lugo, 5 años en Vigo, 3 años en Coruña y Viveiro y 2 en Verin y Trives, abarcarcando 40 registros anuales.

Para la recogida de las muestras se instaló en zonas cercanas al centro de cada una de las ciudades, un captador modelo Lanzoni VPPS-2000. Este sistema de captación no viable, permite conocer la concentración atmosférica de esporas, incluso hora a hora y referirlas a un volumen de aire. La metodología utilizada ha sido la propuesta por la Red Española de Aerobiología (Domínguez et al., 1992).

Para la identificación de las mitosporas se ha

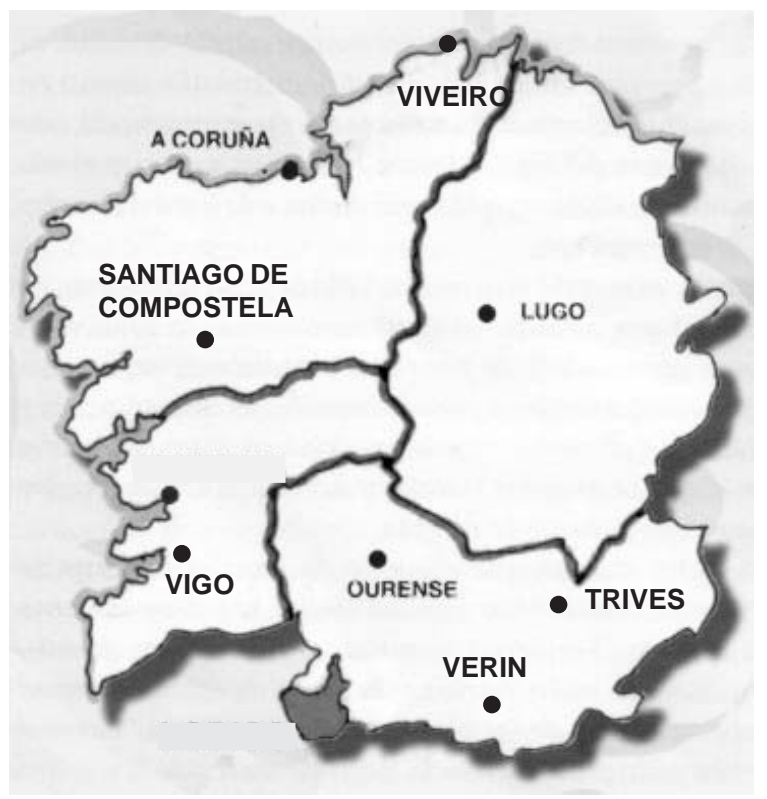

Figura 1.- Localización de las estaciones aerobiológicas en Galicia (España)

seguido el trabajo de Mediavilla et al. (1997), quienes teniendo en cuenta la morfología de las mismas al microscopio óptico y atendiendo a las especies más comunes en nuestra área, diferencian dos tipos:

a) C.cladosporioides, que incluye a C. cladosporioides (Fr.) de Vries, C. avellaneum de Vries, C. elatum (Harz) Nannf., C. oxysporum Berk \& Curt, entre otras especies. Presentan mitosporas unicelulares, de color pardo oliváceo, con cicatrices oscuras en su base indicando el lugar de unión en la cadena. Su forma varía entre elipsoidal a limoniforme y sus paredes son lisas o débilmente rugosas.

b) C. herbarum, incluye a C. herbarum (Pers.) Link ex Gray, a C. sphaerospermum Penz. y a C. macrocarpum Preuss, entre otras especies. Presentan mitosporas unicelulares de extremos redondeados aunque con frecuencia son septadas, dependiendo de la fase de maduración, de color pardo dorado, con cicatrices protuberantes de color pardo, elipsoidales o cilíndricas y claramente rugosas.

\section{RESULTADOS}

Partiendo del análisis de los 40 registros anuales, se observó una mayor concentración de mitosporas de Cladosporium en la atmósfera de las localidades del interior de Galicia, las mayores concentraciones promedio se obtuvieron en Trives (326.898), Verín (289.169) y Ourense (208.098) en comparación con las situadas en la costa (Coruña, Viveiro, Vigo) (Tabla 1). Al analizar de forma independiente los valores promedio del período de 
Tabla 1.- Total y promedio de mitosporas de Cladosporium en las ocho localidades gallegas

\begin{tabular}{|c|c|c|c|c|c|c|c|c|}
\hline Año & Ourense & Santiago & Coruña & Viveiro & Lugo & Vigo & Verin & Trives \\
\hline $\mathbf{1 9 9 3}$ & 113.368 & --- & --- & --- & --- & --- & --- & --- \\
\hline $\mathbf{1 9 9 4}$ & 201.326 & --- & --- & --- & --- & --- & --- & --- \\
\hline $\mathbf{1 9 9 5}$ & 185.541 & --- & --- & --- & --- & --- & --- & --- \\
\hline $\mathbf{1 9 9 6}$ & 220.990 & --- & --- & --- & --- & --- & --- & --- \\
\hline $\mathbf{1 9 9 7}$ & --- & 90.533 & --- & --- & --- & --- & --- & --- \\
\hline $\mathbf{1 9 9 8}$ & --- & 232.135 & --- & --- & --- & --- & --- & --- \\
\hline $\mathbf{1 9 9 9}$ & --- & 138.761 & --- & --- & --- & --- & --- & --- \\
\hline $\mathbf{2 0 0 0}$ & 122.999 & 150.008 & --- & --- & --- & 83.499 & --- & --- \\
\hline $\mathbf{2 0 0 1}$ & 277.833 & 138.789 & 79.725 & 65.203 & 196.092 & 77.153 & --- & --- \\
\hline $\mathbf{2 0 0 2}$ & 355.093 & 148.011 & 125.037 & 166.716 & 186.176 & 203.315 & 352.490 & 344.101 \\
\hline $\mathbf{2 0 0 3}$ & 240.294 & 116.285 & 85.417 & 149.589 & 153.469 & 97.236 & 225.848 & 309.694 \\
\hline $\mathbf{2 0 0 4}$ & 138.956 & 90.464 & --- & --- & 88.586 & 56.294 & --- & --- \\
\hline $\mathbf{2 0 0 5}$ & 224.579 & 131.361 & --- & --- & 137.851 & 157.214 & --- & --- \\
\hline Promedio & 208.098 & 137.594 & 96.726 & 127.169 & 152.435 & 112.452 & 289.169 & 326.898 \\
\hline
\end{tabular}

estudio de cada especie, se observa que las de $\boldsymbol{C}$. cladosporioides son más abundantes en Santiago, Coruña, Viveiro y Vigo, las de $\boldsymbol{C}$. herbarum destacan en Lugo, Verín y Trives, mientras que en Ourense la proporción es similar. Expresado en porcentajes, significa que $\boldsymbol{C}$. cladosporioides representa un 49,7\% en Ourense, un 59.4\% en Santiago, un $74.2 \%$ en Coruña y un $58.2 \%$ en Vigo, mientras que las $\boldsymbol{C}$. herbarum representan un $57.4 \%$ en Lugo, un 63.9\% en Verín y un 70\% en Trives (Figura 2).

En cuanto a su abundancia en la atmósfera a lo largo del año, el período de esporulación principal (P.E.P.) de ambos tipos coincide en la época estival, principalmente entre los meses de julio a septiembre. Las concentraciones medias diarias durante este período oscilaron entre valores de 515 mitosporas $/ \mathrm{m}^{3}$ en Santiago y 295 mitosporas $/ \mathrm{m}^{3}$ en Vigo para Cl. cladosporioides y de 1.197 mitosporas/ $\mathrm{m}^{3}$ en Trives a 133 mitosporas $/ \mathrm{m}^{3}$ en Coruña para $\boldsymbol{C}$.

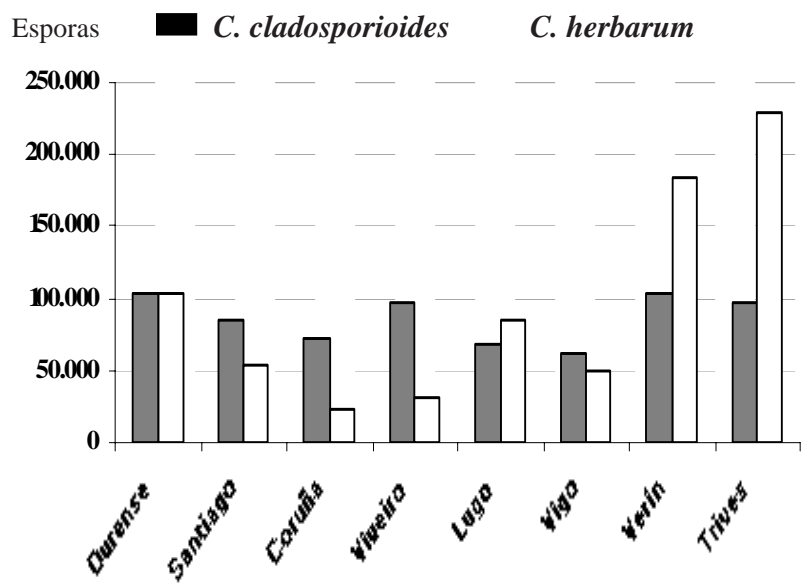

Figura 2.- Representatividad de las mitosporas de $C$. cladosporioides y $C$. herbarum en las 8 localidades

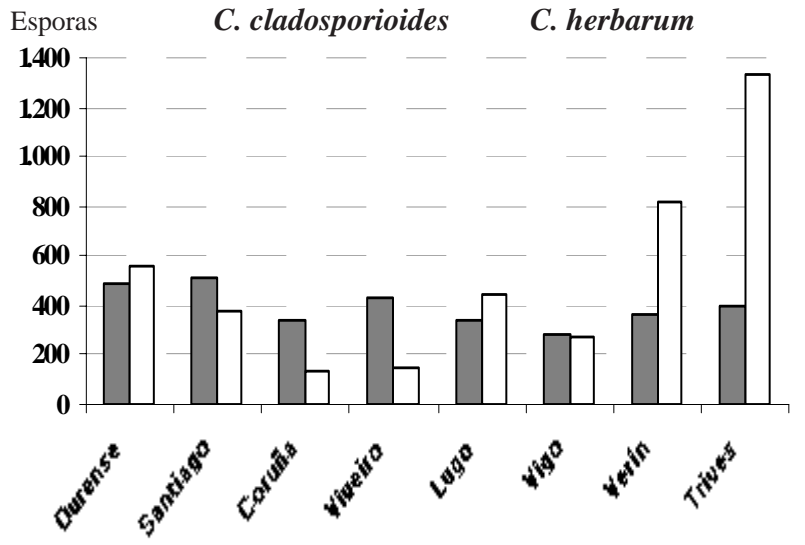

Figura 3.- Valores promedio de las concentraciones medias diarias de mitosporas de C.cladosporioides y C. herbarum durante el P.E.P.

herbarum, con valores intermedios del intervalo señalado para las demás localidades (Figura 3).

Las concentraciones máximas diarias de mitosporas de ambas especies también se localizan en verano, con algunas excepciones. El máximo diario más elevado de C. cladosporioides de las ocho localidades se registró en Ourense, con 7.556 mitosporas $/ \mathrm{m}^{3}$ el 8 se septiembre del año 2004, mientras que el más elevado de $\boldsymbol{C}$. herbarum fue de 7.130 mitosporas $/ \mathrm{m}^{3}$ el 8 de julio del año 2003 en Trives (Tabla 2).

Finalmente, se ha realizado el estudio del comportamiento intradiario con el fin de conocer si hay alguna variación de la concentración de mitosporas a lo largo del día (Figuras 4 y 5), resultando que en la mayoría de las localidades se observa un incremento de la concentración durante la tarde-noche, como ocurre por 


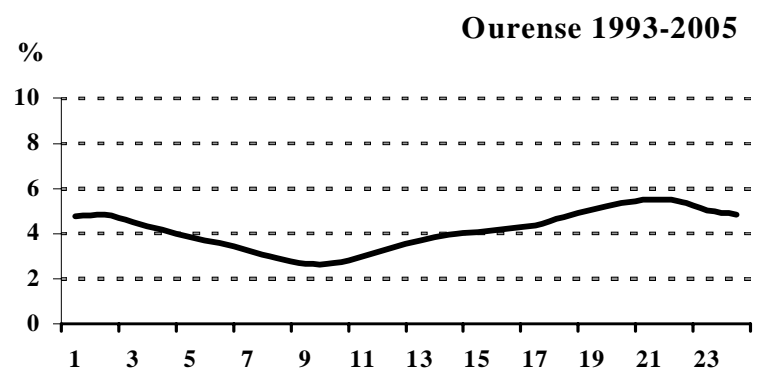

Coruña 2001-2003

$\%$

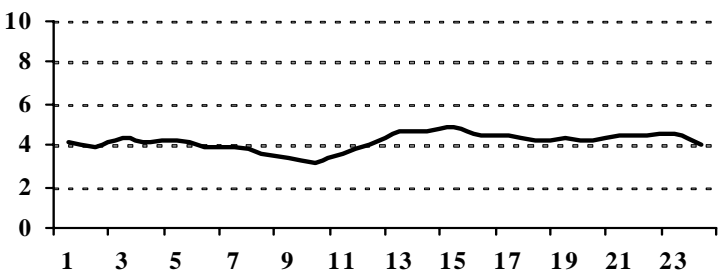

Lugo 2001-2005

$\%$

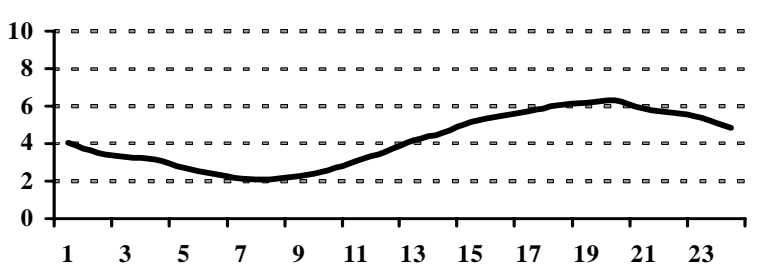

Verin 2002-2003

$\%$

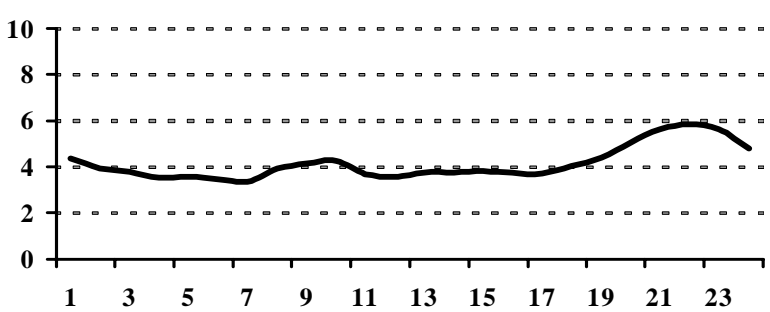

Santiago 1997-2005

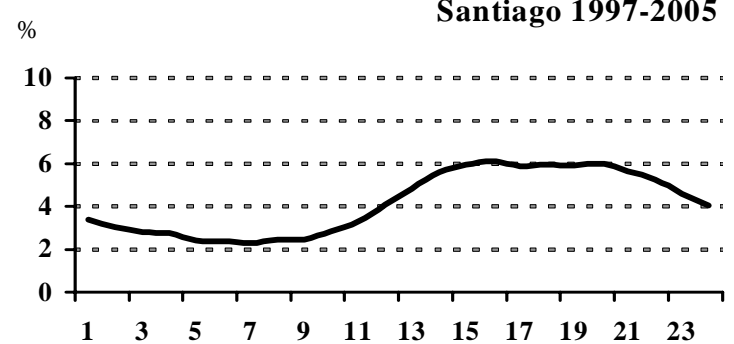

Viveiro 2001-2003

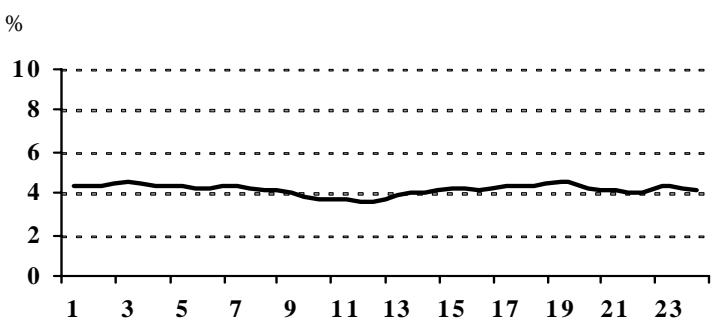

Vigo 2000-2005

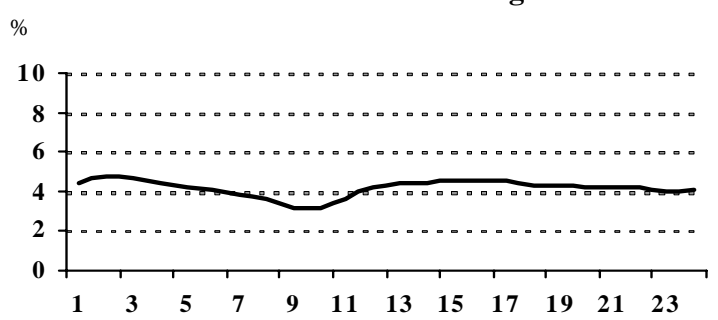

Trives 2002-2003

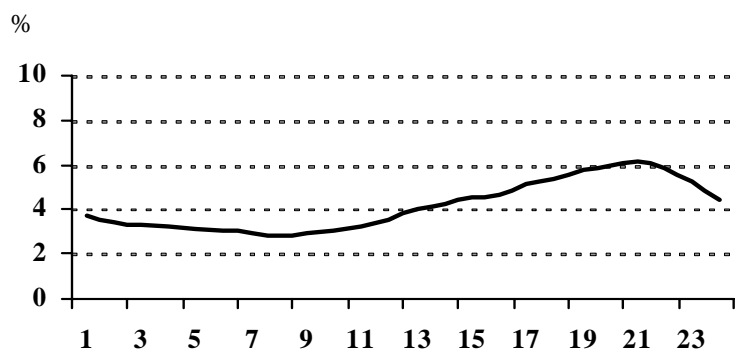

Figura 4.- Variación intradiaria de Cl.cladosporioides en las 8 localidades

Tabla 2.- Concentraciones máximas diarias de las mitosporas de C. cladosporioides y C. herbarum en las ochos localidades

\begin{tabular}{cccccccc} 
Ourense & Santiago & Coruña & Viveiro & Lugo & Vigo & Verín & Trives \\
\hline \multicolumn{7}{c}{ Cladosporium cladosporioides } \\
\hline $\mathbf{7 . 5 5 6}$ & $\mathbf{5 . 9 5 1}$ & $\mathbf{3 . 9 4 4}$ & $\mathbf{3 . 6 4 7}$ & $\mathbf{4 . 5 1 0}$ & $\mathbf{5 . 6 7 8}$ & $\mathbf{6 . 6 9 4}$ & $\mathbf{4 . 7 5 6}$ \\
$8 / 9 / 04$ & $1 / 7 / 01$ & $24 / 8 / 02$ & $11 / 7 / 03$ & $9 / 7 / 01$ & $15 / 9 / 02$ & $21 / 1 / 02$ & $12 / 9 / 02$ \\
\hline \multicolumn{7}{c}{ Cladosporium } & herbarum \\
\hline $\mathbf{7 . 0 7 1}$ & $\mathbf{4 . 0 7 4}$ & $\mathbf{1 . 6 0 9}$ & $\mathbf{3 . 6 9 4}$ & $\mathbf{4 . 2 3 7}$ & $\mathbf{6 . 4 7 2}$ & $\mathbf{6 . 3 5 7}$ & $\mathbf{7 . 1 3 0}$ \\
$24 / 6 / 95$ & $10 / 7 / 99$ & $11 / 7 / 03$ & $11 / 7 / 03$ & $6 / 7 / 01$ & $18 / 8 / 02$ & $10 / 7 / 02$ & $8 / 7 / 03$
\end{tabular}




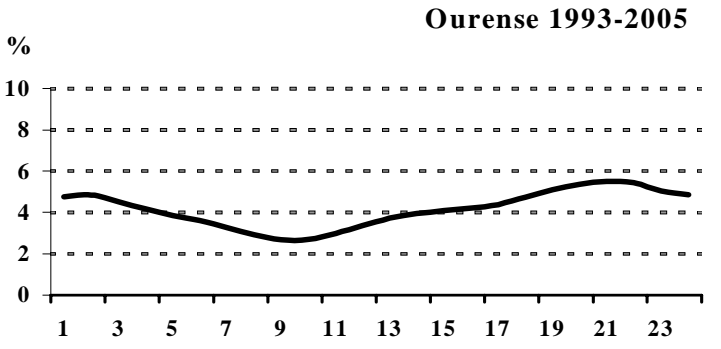

Coruña 2001-2003

$\%$

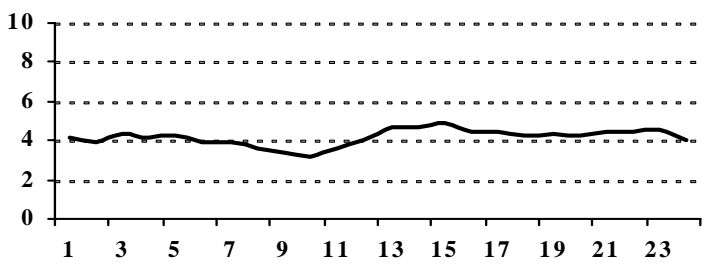

Lugo 2001-2005

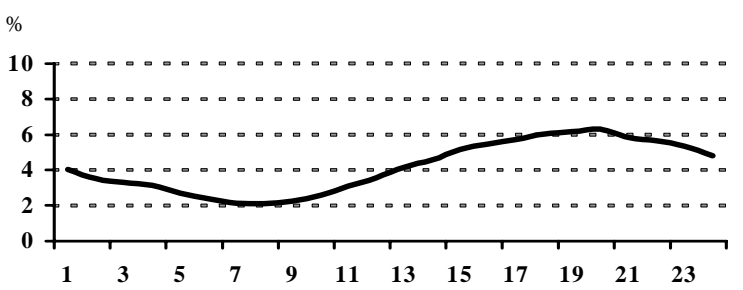

Verin 2002-2003

$\%$

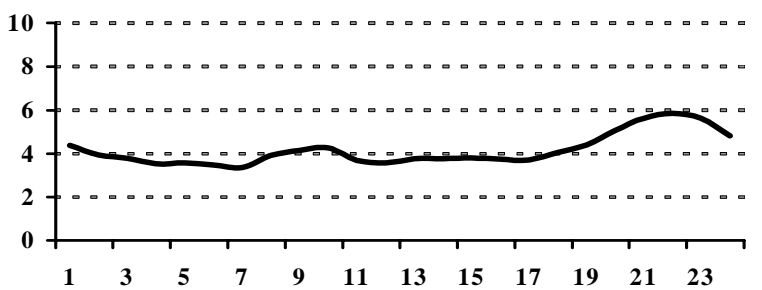

Santiago 1997-2005

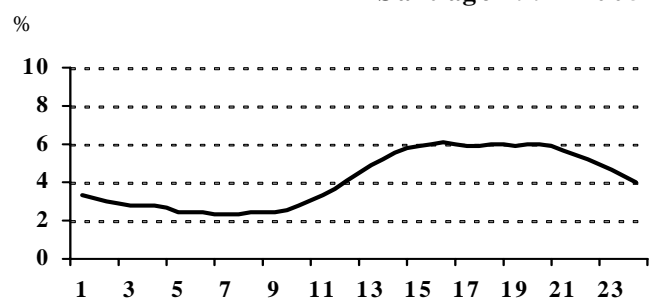

Viveiro 2001-2003

$\%$

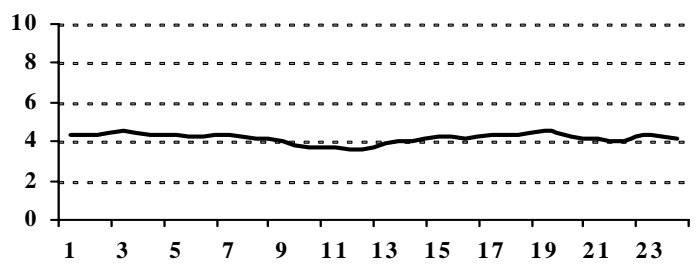

Vigo 2000-2005

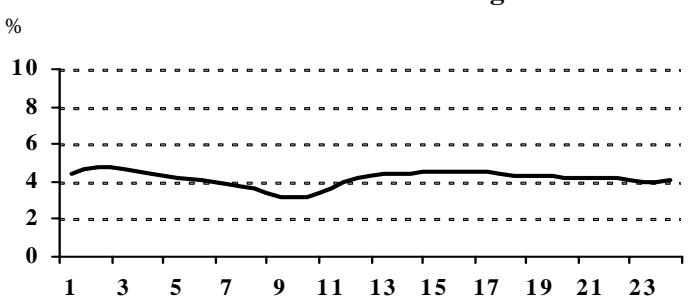

Trives 2002-2003

$\%$

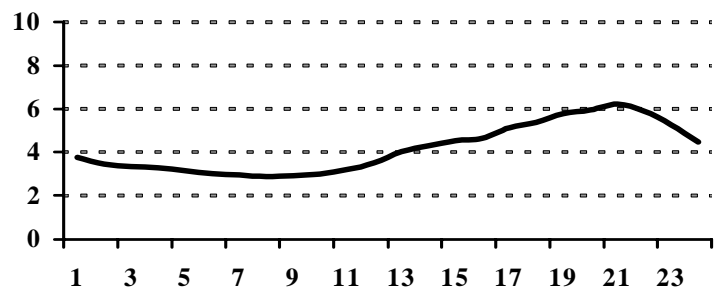

Figura 5.- Variación intradiaria de C. herbarum en las 8 localidades

Tabla 3.- Promedio del número de días con diferentes concentraciones de mitosporas de Cladosporium

\begin{tabular}{c|ccc} 
& $>\mathbf{5 0 0}$ & $>\mathbf{1 . 0 0 0}$ & $>\mathbf{3 . 0 0 0}$ \\
\hline Ourense & 113 & 64 & 11 \\
Santiago & 79 & 42 & 5 \\
Coruña & 62 & 27 & 2 \\
Viveiro & 87 & 40 & 3 \\
Lugo & 95 & 46 & 5 \\
Vigo & 63 & 30 & 4 \\
Verín & 142 & 94 & 20 \\
Trives & 151 & 112 & 24 \\
\hline
\end{tabular}

ejemplo en Santiago con $\boldsymbol{C}$. cladosporioides o en Trives con $\boldsymbol{C}$. herbarum. En otras localidades la concentración máxima se adelanta a las 13-14 h (Santiago y Lugo para C.herbarum) o muestran variaciones poco marcadas (Coruña y Viveiro para $\boldsymbol{C}$. cladosporioides).

La ciudad que presentó el mayor número promedio de días al año con altas concentraciones de mitosporas fue Trives, seguido de Verín y Ourense (Tabla 3).

\section{DISCUSION}

Las diferencias cuantitativas en la concentración anual de mitosporas en las ocho localidades de estudio, 
pueden ser debidas a las condiciones biogeográficas de cada área y a las condiciones meteorológicas de cada año, ya que ambas causas influyen en la disponibilidad de un sustrato adecuado para el desarrollo de los hongos y favorecen o dificultan su esporulación.

Estudios anteriores realizados en Galicia, han señalado una mayor concentración de mitosporas en el interior de la región en comparación con las ciudades costeras (Hervés, 2005). Este argumento parece adecuado incluso para explicar porque la atmósfera de Coruña, una ciudad prácticamente rodeada por el mar, registra el promedio más bajo (97.681 mitosporas) frente a Vigo (114.312 mitosporas) o Vivero (128.730 mitosporas), situadas en una zona más protegida de la costa y con un área de vegetación circundante mucho mayor. En cuanto a la influencia del clima, diversos autores han señalado el efecto positivo de la temperatura y negativo de la humedad y las precipitaciones en la concentración atmosférica de mitosporas de Cladosporium (Mediavilla et al., 1997; Sabariego et al., 2004). Por lo general, se pone de manifiesto que para la liberación de los conidios de estos hongos a la atmósfera, es necesario un cierto grado de sequedad ambiental, que se obtiene cuando aumenta la temperatura (Corden et al., 2003).

Atendiendo a las referencias bibliográficas de otros autores que utilizan la misma metodología de captación (método volumétrico no viable), se confirma la importancia de Cladosporium a nivel mundial. Los promedios anuales de Cladosporium son muy elevados en distintas localidades del sur de España, del orden de 347.448 mitosporas en Córdoba (Mediavilla et al., 1997) y 252.069 mitosporas en Granada (Sabariego et al., 2004), mientras que en Almería, Tarragona y Madrid, no alcanzan las 100.000 (Belmonte \& Roure, 2002; Sáenz \& Gutiérrez, 2003). En distintos países europeos, también las mitosporas de Cladosporium tienen una elevada representatividad en la atmósfera, en Holanda, pueden alcanzar concentraciones de 700.000 al año (Nikkeels et al., 1996).

Cladosporium (junto con Alternaria) son los géneros más frecuentes en la India, donde Jothish \& Nayar (2004) señalan que Cladosporium puede alcanzar el 44,7\%. También en distintas ciudades de China (Yang et al., 1989) y Egipto, donde Youssef \& Karma (1988) señalan a Cladosporium con un 39,6\% o en Melbourne, donde representa hasta un 41,7\% (Mitakakis \& Guest, 2001). Lo mismo ocurre en el continente americano, destacando los elevados niveles en Jamaica (Royes, 1987). Cladosporium representa un $80 \%$ del total de esporas en Corpus Christi, Texas (Dixit et al., 2000) y un 32\% en Caxis do Sul, Brasil (Antoni de et al., 2004).

Con respecto a su abundancia estacional, el aumento paulatino de mitosporas de Cladosporium se produce en las estaciones gallegas desde el mes de mayo y se mantiene durante el verano, mientras las concentraciones bajas en los meses de invierno, coinciden con otras localidades españolas, como León (Vega et al., 2000) y Tarragona (Belmonte \& Roure, 2002). Por el contrario, en localidades mas meridionales la distribución a lo largo del año, sigue un modelo bimodal con dos períodos por lo general bien definidos, uno localizado en primavera y otro en otoño (Galán et al., 1998; Sabariego et al., 2004). Estas diferencias cuantitativas, pueden estar relacionadas con la temperatura, que es uno de los factores determinantes para el desarrollo fúngico y la esporulación, por ello, aquellas situadas en el sur peninsular muestran sus máximos mensuales en una época más temprana que las del norte. En distintas ciudades europeas las concentraciones más elevadas de mitosporas de Cladosporium se encuentran en los meses de verano (Corden et al., 2003). En Pozna (Polonia) y en Londres, son frecuentes en la atmósfera desde mediados de mayo (Emberlin et al., 1995; Stach et al., 2003); en Praga, el máximo se localiza en el mes de julio (Hysek et al., 1991) y en Italia se registran elevadas concentraciones en julio y agosto (Picco, 1992). En áreas situadas en latitudes bajas, que presentan un clima tropical o desértico, la temperatura no es un factor tan influyente como la precipitación y la humedad, por lo que Cladosporium parece evitar los meses veraniegos y tiende a aparecer en los meses previos o posteriores a esta estación, como ocurre en Egipto, donde su abundancia es más elevada en invierno que en verano (Yousseff \& Karma, 1988).

Las concentraciones máximas diarias de Cladosporium registradas en las localidades gallegas, con valores cercanos a las 7.000 mitosporas $/ \mathrm{m}^{3}$ fueron ampliamente superadas en otras ciudades españolas, como en Córdoba (49.536 mitosporas $/ \mathrm{m}^{3}$ el 17 de septiembre de 1996), en Salamanca (22.500 mitosporas $/ \mathrm{m}^{3}$ el 29 de junio de 1995) y en Tenerife (14.319 mitosporas $/ \mathrm{m}^{3}$ el 1 de noviembre de 1988) (Mediavilla et al., 1997; Domínguez et al., 1992; Pérez et al., 2002).

Finalmente, en cuanto a la distribución intradiaria y de forma independiente de la localidad, las mitosporas de Cladosporium presentan una marcada tendencia a la dispersión diurna. Diversos autores señalan la relación de elevadas concentraciones de esporas con temperaturas elevadas, ya que facilitan el mecanismo de dispersión mecánica de los propágulos. Atluri et al. (2002), indican que el modelo obtenido depende de la localización geográfica, así como del clima del área y del emplazamiento del captador. En ciudades del sur de España, como en Granada o Córdoba, los modelos de variación de $\boldsymbol{C}$. cladosporioides coinciden en presentar una mayor concentración por la tarde, mientras las de $\boldsymbol{C}$. herbarum abundan ya desde finales de la mañana y se mantienen con valores elevados por la tarde, con máximos 
a las 13h -14h (Mediavilla et al., 1997), coincidiendo con los resultados obtenidos en Ourense, Lugo o Santiago. Por el contrario, en ciudades como Almería, la representación atmosférica es mucho más homogénea y con escasas oscilaciones a lo largo del día (Sabariego et al., 2004), coincidiendo con los resultados obtenidos para Coruña o Viveiro.

Con el fin de valorar la incidencia clínica que las mitosporas de Cladosporium pueden causar en la población sensibilizada, algunos autores han señalado niveles diarios del orden de 3.000/ $\mathrm{m}^{3}$ (Gravesen, 1979; Caretta, 1992), mientras que otros consideran que valores superiores a 100 esporas $/ \mathrm{m}^{3}$, son suficientes para que comiencen los primeros síntomas alérgicos (Ballero et al., 2000).

Teniendo en cuenta estos criterios, las localidades gallegas con un ambiente con mayor riesgo de incidencia alérgica para las personas asmáticas serían : Ourense, Verín y sobre todo Trives ya que alcanza 151 días de promedio con valores superiores a 500 esporas $/ \mathrm{m}^{3} /$ día, 112 días con $>1.000$ esporas $/ \mathrm{m}^{3} /$ día y 24 días con más de 3.000 esporas/ $\mathrm{m}^{3} /$ día.

\section{REFERENCIAS}

Aira, M.J.; Rojas, T. \& Jato, V. (2002). Fungi associated with three houses in Havana (Cuba). Grana, 41: 114-118

Airaudi, D. \& Marchisio, V.F. (1997). Fungal biodiversity in the aire of Turin. Mycopathologia, 136 (2): 95-102

Albelda, Y.; Rodríguez-Rajo, F.J.; Jato, V. \& Aira, M.J. (2005). Concentraciones atmosféricas de propágulos fúngicos en viñedos del Ribeiro (Galicia. España). Boletin Micológico 20: 1-8

Antoni de, B.C.; Fernández, D.; Vergamini, S.M. \& Valencia, R. (2004). Predominating fungal spores in atmospheric aerosol in the city of Caxis do Sul, Rio Grande do Sul, Brazil during 2001 and 2002. XI International Palynological Congress. Granada. pág. 366

Atluri, J.B. \& Murthy, D.V. (2002). Effect of harvesting operations on fungal spore populations in air. J. Environ. Biol., 23 (1): 65-69

Ballero, M.; Piu, G. \& Ariu, A. (2000). The impact of the botanical gardens on the aeroplankton of the city of Cagliari, Italy. Aerobiologia, 16: $143-147$

Belmonte, J. \& Roure, J.M. (2002). Aerobiología en Catalunya: estación de Tarragona (2000-2001). REA, 7: 161-166

Caretta, G. (1992). Epidemiology of allergic disease: the fungi. Aerobiologia, 8: 439-445

Chakraborty, P.; Gupta, S.; Chowdhury, I.; Majumdar, M. \& Chanda, S. (2001). Differences in concentrations of allergenic pollens and spores different heights on an agricultural farm in West Bengal, India. Ann. Agric. Environ. Med., 8 (2): 123-130

Corden, J.M.; Forster, G. \& Newlands, M. (2003). Were fungal spores responsible for the increased asthma admissions in Derby, England after severe thunderstorms on July $30^{\text {th }}$ 2002? Third European Symposium in Aerobiology. Worcester. U.K. pág. 22

D’Amato, G. \& Spieksma, F.Th.M. (1995). Aerobiologic and clinical aspects of mould allergy in Europe. Allergy, 50: 870-77

Dale, R.E.; Cakmak, S.; Burnett, R.T.; Judek, S.; Coates, F. \& Brook, J.R. (2000). Influence of ambient fungal spores on emergency visits for asthma to a regional children's Hospital. Am. J. Respir. Crit. Care. Med., 162 (6):2087-2090

Dixit, A.; Lewis, W.; Baty, J.; Crozier, W. \& Wedner, J. (2000). Deuteromycete aerobiology and skin-reactivity patterns. A two year concurrent study in Corpus Christi, Texas, U.S.A. Grana, 39: 209218

Domínguez, E.; Galán, C.; Villamandos, F. \& Infante, F. (1992). Manejo y evaluación de los datos obtenidos en los muestreos aerobiológicos. Monografías R.E.A./E.A.N., 1: 1-13

Emberlin, J.; Newman, T. \& Bryanta, R. (1995). The incidencie of fungal in the ambient air and inside homes: evidence from London. Aerobiologia, 11: 253-258

Galán, C.; Cariñanos, P.; Alcázar, P.; Infante, F.; Castro, A. \& Domínguez E. (1998). Aerobiología en Andalucía: estación de Córdoba (1997). REA, 4: 21-24

Gavesen, S. (1979). Fungi as a cause of allergic disease. Allergy, 34 (3): $135-154$

Hervés, M. (2005). Estudio aerobiológico en cuatro localidades gallegas. Tesis Doctoral. Univeridad de Santiago

Hysek, J.; Fisar, Z. \& Binek, B. (1991). Lon-run monitoring of bacteria, yeast and other micromycetes in teh air of an industrial conurbation. Grana, 29: 450-453

Jothish, P.S. \& Nayar, T.S. (2004). Airborne fungal spores in a sawmill environment in Aplacad district, Kerala. India. Aerobiologia, 20: 7581

Maggi, O.; Persiani, A.M.; Gallo, F.; Valenti, P.; Pasquariello, G.; Sclocchi, M.C. \& Scorrano, M. (2000). Airborne fungal spores in dust present in archives: proposal for a detection method, new for archival materials. Aerobiologia, 16: 441-448

Mediavilla, A.; Angulo, J.; Infante, F. \& Domínguez, E. (1997). Influence of meteorological factors on the incidence of Cladosporium Link ex Fr. conidia in the atmosphere of Córdoba (Spain). Phenology in seasonal climates, I: $117-126$

Mitakakis, T.Z. \& Guest, D.I. (2001). A fungal spore calender for the atmosphere of Melbourne, Australia for the year 1993. Aerobiologia, 17: $171-176$

Montacutelli, R.; Maggi, O.; Tarsitani, G. \& Gabrielli, N. (2000.) Aerobiological monitoring of the «Sistine Chapel»: airborne bacteria and microfungi trends. Aerobiologia, 16: 441-448

Myszkowska, D.; Stepalska, D.; Obtulowicz, K. \& Porebski, G. (2002). The relationship between airborne pollen and fungal spore concentrations and seasonal pollen allergy symptoms in Cracow in 1997-1999. Aerobiologia, 18: 153-161

Nikkels, A.H.; Terstegge, P. \& Spieksma, F.Th.M. (1996). Ten types 
of microscopically identificable airborne fungal spores at Leiden. The Netherlands. Aerobiologia, 12: 107-112

Noé, A.; Gutierrez, M.; Diez,A. \& Sabariego, S. (2006). Aerobiología de los 10 tipos de esporas fúngicas más frecuentes en la atmosfera de Madrid. Polen 16. 118-119

Oliveira, M.; Ferreira, A; Abreu, I.; Ribeiro, H.; Machado, H. \& Reis, A.(2006). Atmospheric fungal spore counts and emergency admissions with respiratory diseases in a regional hospital. Polen 16: $123-124$

Pérez, P.; Rodríguez, D.; Suárez, R. \& Sánchez, J. (2002). Variación anual de esporas en la atmósfera de Salamanca durante los años 1995 y 2000. XIV Simposio de A.P.L.E, Salamanca. España

Picco, A.M. (1992). Presence in the atmosphere of vine and tomato pathogens. Aerobiologia, 8: 459-463

Rodríguez, F.J.; Seijo, M.C. \& Jato, V. (2002). Estudio de los niveles de fitopatógenos para la optimización de cosechas de Vitis vinífera en Valdeorras (1998). Botánica Complutensis, 26: 121-135

Royes, V.I.J. (1987). Some components of the air spora in Jamaica and their posible medical application. Grana, 26: 1551-157

Sabariego, S.; Díaz de la Guardia, C. \& Alba, F. (2004). Incidencia de esporas de Alternaria y Cladosporium en la atmósfera de la ciudad de Almería (SE de España): relación con los parámetros meteorológicos. R.I.A.M
Sáenz, C. \& Gutiérrez, M. (2003). Esporas atmosféricas en la Comunidad de Madrid. Documentos Técnicos de Salud Pública, 83. Instituto de Salud Pública. Madrid

Stach, A.; Josefiak, M.; Filipiak, M.; Sekulska, M.; Knopkiewicz, M.; Szyszka, A.; Czarnecka, M. \& Silny, W. (2003). Results of first interdisciplinary studies of indoor and aoutdoor micoorganism concentration in Pozna, Polan 2002. Third European Symposium in Aerobiology. Worcester. U.K. pág. 120

Vega, A.; Fernández, D.; Valencia, R.M.; Fernández, A.B.; Santos, F. \& Latasa, M. (2002). Aerobiología en Castilla y León: estación de León (2000-2001). REA, 7: 119-124

Vijay, H.M; Burton, M.; Young, N.M.; Copeland, D. \& Corlett, M. (1991). Allergenic componets of isolates of Cladosporium herbarum. Grana, 30: 161-165

Yang, J.P.; Yin, Q.Z.; Ye, M.J.; Zhang, C.W. \& Yu, Q.A. (1989). Quantitative study of airborne fungi at three functional section of Chengdu city. Ниа Xi Yi Ke Da Xue Bao, 20 (4): 448-451

Youssef, Y.A. \& Karma, E.D. (1988). Airborne spores of opportunistic fungi in the atmosphere of Cairo, Egypt. Grana, 27: 89-92 